\title{
Über Bau und Entwicklung der Spermien von Rana fusca.
}

\author{
Von
}

Professor Dr. Ivar Broman, Lund.

Hierzu Tafel XIX, XX und 4 Textfiguren.

\section{Einleitung.}

Die diesem Aufsatz zu Grunde liegenden Untersuchungen wurden grösstenteils schon vor 6 Jahren ausgeführt. Das hierzu benutzte Material war indessen in einer etwas späten Jahreszeit (September und Oktober) konserviert worden, und zeigte daher aus den allerersten Entwickelungsstadien nur eine relativ geringe $Z a h l$ von Spermatiden, 'und an diesen war das Verhalten des Idiozoms nicht gut zu beobachten. Obgleich ich nun im übrigen die Umwandlung der Spermatiden in Spermien klarstellen konnte, und obgleich hier keine wichtigere Abweichungen des Idiozoms zu erwarten waren, verschob ich die ausführliche Publikation meiner Ergebnisse in der Absicht, dieselben in einem folgenden Jahre durch Untersuchung von Juliund August-Material $\mathrm{zu}$ vervollständigen.

Indessen wurde ich durch andere Aufgaben verhindert, dieses Thema weiter zn behandeln, und beschränkte mich daher darauf, den Bau der reifen Spermien von drei Ranaarten und von einigen anderen Amphibien in einem schwedischen Aufsatz (B roman 1900: 2) und die wichtigsten Züge der Spermiogenese bei Rana fusca in einer kurzen Figurenerklärung (B r o m a $\mathrm{n}$ 1901: 1) vorläufig mitzuteilen.

Diese Angaben, ebenso wie die dazu gehörenden Textfguren, wurden von Meves (1902) in seinem bekannten kritischen Referat ,Struktur und Histogenese der Spermien" fast in extenso wiedergegeben. Ich hielt es daher nicht mehr für nötig, meine schwedisch geschriebene Arbeit in Deutsch erscheinen zu lassen.

Auf die ausführlichere Schilderung der Rana-Spermiogenese war es nun meine Absicht zu verzichten, bis ich Gelegenheit gehabt bätte, die Spermiogenese mehrerer Batrachier komparativ zu untersuchen.

Indessen ist neulich eine Arbeit von Retzius (1906) über den Bau der Amphibienspermien erscbienen, welche zwar in den meisten Punkten meine Beobachtungen über die Ranaspermien bestätigt, in einigen Punkten aber zu wesentlich anderen Resultaten kommt. Diese Divergenz bildet nun für mich die Veranlassung, meine alten Beobachtungen sofort zu veröffentlichen. Ich glaube nämlich durch die Beschreibung der Spermiogenese bei Rana fusca beweisen zu können, dass die von mir gegebene Schilderung über den Bau der reifen Ranaspermien in allen Punkten richtig ist.

Betreffs des Baues der reifen Spermien bei den anderen von mir untersuchten Batrachiern (Bufo, Hyla, Bombinator und Pelobates) hat Retzius 
(1906) ebenfalls im allgemeinen meine Befunde bestätigen können. In einigen wichtigen Punkten (speziell betreffs der Spermien von Hyla, Bufo und Pelobates) weichen indessen auch hier die Beobachtungen dieses Autors beträchtlich von den meinigen ab. Wer von uns hier im Unrecht ist, müssen kommende Nachuntersuchungen lehren, welche die Entwickelung der betreffenden Spermien genau verfolgen. Dieses ist aber - meiner Erfahrung nach - nur an Hodenschnittpräparationen möglich. Die in Ausstrichspräparaten sichtbaren Entwickelungsstadien der Spermien haben - glaube ich -, wenn sie allein dafür benutzt werden, für ein genaues Studium der Spermiogenese nicht viel Wert.

\section{Ueber den Bau der Rana-Spermien.}

\section{Frühere Untersuchungen.}

Die Spermien des Frosches wurden schon von Leeuwenhoek (1687) beschrieben und abgebildet (vgl. Textfig. 1-3) und zwar „als geschlängelte Tierchen mit einem dicken Vorderteil und spitzen Schwanz. "1) Etwa 100 Jahre später wurden sie aber von

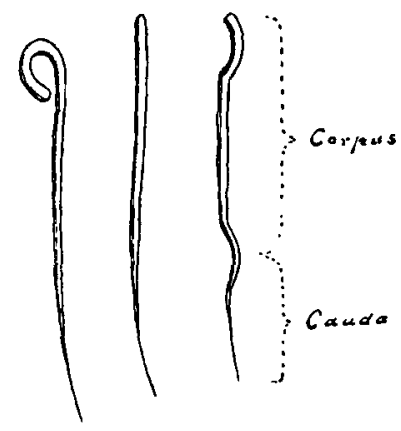

Fig. 1-3.

(Nach Leeuwenhoek, 1687.)

Froschspermien. Fig. 1 (mit umgebogenem Kopfe) und Fig. 2 (gestrecikt) tot; Fig. 3 lebend.

Gleichen (1788) als schwanzlose Bildungen abgebildet. Auch Treviran us (1816) konnte an den Froschspermien keinen Schwanz unterscheiden (vgl. Textfig. 4:1) und behauptet daher, Lee uwe nhoek habe sich diese Spermien „nach seiner Phantasie vorgestellt."

Prévost und Dumas (1824) bilden sie wieder als mit Kopf und Schwanz versehene Bildungen ab (Textfig. 4: 2). Die

1) Cit. nach Treviranus (1816). 
betreffenden Bilder stimmen aber gar nicht mit den von Le euw enhoek gegebenen überein; und ich finde es glaubhaft, dass auch diese Autoren den wabren Schwanz der Froschspermien nicht gesehen baben. Noch 1833 bildet $\mathrm{Czermak}$ die Froschspermien als schwanzlose Bildungen ab (T'extfig. 4: 3).

Erst im Jahre 1847 wurde der Schwanz der Froschspermien wieder und zwar von Kölliker entdeckt. Dieser Autor bildet die Spermien von Rana temporaria (= fusca) als aus einem recht langen, nach vorn zugespitzten Kopf und einem sehr feinen Schwanzfaden bestehend ab. Der letztgenannte war - bei noch

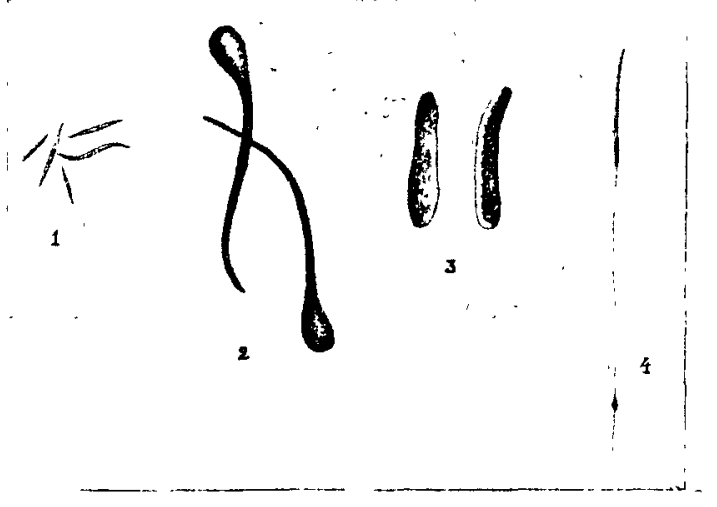

Fig. 4.

Froschspermien :

1. nach Treviranus (1816)

2. $\gg$ Prévost und Dumas (1824)

3. "Czermak (1833)

4. Kö $11 \mathrm{iker}(18 \pm 7)$.

nicht ganz reifen Spermien - nahe an dem freien Ende mit einem kleinen Knötchen versehen (Textfig. 4: 4).

Wagner und Leuckart (1852) und Ecker (1851-59) bilden die Spermien desselben Tieres in etwa ahnlicher Weise ab, jedoch ohne Schwanzknötchen und mit weniger spitzem Kopfe.

R emak (1854) hebt indessen die oben erwähnten Schwanzknötchen als den reifen Spermien von Rana temporaria regelmässig zukommende Bildungen hervor. Diese Körnchen zeigten sehr lebhafte Formveränderungen ,wie eine Amoeba " und wurden von Remak als ein Analogon zu der undulierenden Membran der Salamanderspermien betrachtet. 
Die Spermien von Rana esculenta wurden von Schweigger-Seidel (1865) abgebildet und als mit einem vorn stumpf abgerundeten Kopf, einem kurzen, dicken Mittelstück und einem dünnen Schwanztaden verselienen Bildungen beschrieben.

Dass die Spermien von Rana esculenta denjenigen von Rana fusca (= temporaria) sehr unähnlich sind, wird besonders von Leydig (1877) liervorgehoben. Nach diesem Autor ist der Spermienkopf bei Rana esculenta walzenförmig, abgestutzt und etwa doppelt kürzer als der schmalen, vorn scharf zugespitzten Spermienkopf bei Rana fusca. Beiden sollte ein Mittelstück ganz fehlcn. Den Schwanzfaden der betreffenden Spermien bildet er als einen einfachen Faden ab; jedoch bält er „das Vorhandensein einer undulierenden Membran für sehr wahrscheinlich." - Die Spermien von Rana arvalis sind nach Leydig denen von $R$. esculenta sehr ăhnlich und differieren also sehr von den Spermien der Rana fusca; eine Beobachtung, welche später von $\mathrm{Pfl}$ ü ger und $\mathrm{S}$ mith (1883) und B o rn (1883) bestätigt wurde und bekanntlich für die Frage nach der Artberechtigung der Rana arvalis vor allem bestimmend gewesen ist.

v. Ia Valette St. George (1S86) entdeckte am vorderen Kopfende der Spermien von Rana esculenta ein kleines Knöpfchen. Im übrigen bestätigen seine Angaben die Richtigkeit der von Schweigger-Seidel (186ə⿱) gegebenen Beschreibung über diese Spermien. Von dem von Leydig vermuteten Flimmersaume am Scluwanzfaden war keine Spur zu sehen.

Bei den Spermien von Rana fusca wurde das wahre Mittelstück zuerst von v. la Valette St. George (1876) erkannt. (Die von N e umann (1875) unter dem Namen "Mittelstück" beschriebene Partie dieser Spermien ist offenbar mit dem Kopfe identisch. Das von Neumann als Kopf beschriebene Spitzchen muss ein Kunstprodukt gewesen sein). Später ist die Existenz des Mittelstückes bei diesen Spermien von Bertacchini (1891), mir (Broman, 1900: 2) und Retzius (1906) bestätigt worden.

Bertacchini (1891) bildet dasselbe indessen allzu kurz (etwa sechs mal kürzer als den Kopf) ab. Offenbar hat dieser Autor die hintere Grenze desselben, welche, wie schon v. la Valette 
St. Ge orge hervorgehoben hat, von der nackten Schwanzfadenpartie schwer abzugrenzen ist, nicht unterscheiden können.

Selbst habe ich (Broman 1900: 2) bei diesen Spermien immer das Mittelstück (= Retzius' "Verbindungsstück“) etwa halb so lang wie den Kopf gefunden. Bisweilen ist es sogar länger als die halbe Kopflänge, bisweilen aber auch ein wenig kürzer (vgl. Fig. 19-22). Betreffs des feineren Baues des Mittelstückes konnte ich feststellen, dass der eigentliche Schwanzfaden sich in dem Inneren des Mittelstückes unverdickt noch vorn zum Kopfe fortsetzte (vgl. Fig. 16-18) und dass die betreffende Verdickung des Schwanzes durch eine die vordere Schwanzfadenpartie umgebende körnige Hülle hervorgerufen wurde (Fig. 16). In dieser Körnchenhülle, welche kaudalwärts allmählich dünner wurde, konnte ich keine spiralige Anordnung der Körnchen beobachten.

An der Grenze zwischen Mittelstück und Kopfe fand ich zwei grössere unter sich eng verbundene, binter e in a nder gelegene Körner (Fig. 16-18), welche die Verbindung des Schwanzfadens mit dem Kopfe vermittelten. Durch spermiogenetische Untersuchung konnte ich auch feststellen, dass di ese Körner beide Centriolderivate sind, und zwar dass das vordere Korn den vergrösserten proximalen Centriol (= Zentralkörper) und das hintere Korn einen Teil des (ebenfalls vergrösserten) distalen Centriols darstellt. (Vgl. Fig. 1-16.)

An der einen Seite des vorderen Kopfendes fand ich eine von früheren Untersuchern nicht gesehene, mit Gentianaviolett stark färbbare, stabförmige Bildung, welche ich als den Spiess (= das Perforatorium) dieser Spermien (Fig. 16, 19-21) beschrieb.

Meine oben erwähnten Beobachtungen über den Spiess und die Körnchenhülle dieser Spermien sind neulich von Retzius (1906) bestătigt worden. Dagegen hat dieser Autor meine Beobachtungen über die Centriolderivate [ = das Hals st ü ck im Sinne W ald e yers (1901)] und die vordere Schwanzfadenpartie nicht bestätigen können. Er zeichnet die Centriolderivate als zwei nebeneinander gelegene, gleichgrosse Körnchen ab, welche die Verbindung je eines Schwanzfadens mit dem Kopfe vermitteln. Die betreffenden beiden Schwanzfäden, welche in den peripheren Partien des Verbindungsstückes gezeichnet sind (vgl. Retzius (1906) Taf. 23, Fig. 1), sollten sich in der 
kaudalen Partie desselben zu einem einfachen Schwanzfaden verbinden.

Hervorzuheben ist, dass Ret z i u s die betreffenden Spermien, welche offenbar von $R$ ana fusca herstammen, auf einer mit dem Namen Rana esculenta $L$. bezeichneten Tafel abbildet. Die betreffenden Tiere hatte Retzius von einem Tierhändler unter dem Namen R. esculenta bekommen; er halt es aber für möglich, dass dieser Niame unrichtig ist. Ich hebe dieses speziell hervor um weitere Verwechselungen von den so verschiedenen Spermien dieser beiden Ranaarten vorzubeugen. Sogar Retzius hat offenbar diese Verschiedenheit nicht genügend beachtet. Denn er ist der Ansicht, dass man die Spermien der einen Rana-Art als Typus des Genus Rana überhaupt aufstellen kann; und er kritisiert die - meiner Meinung nach - sehr gute Beschreibung Schweigger-Seidels (1865) über den Bau der Spermien von Rana esculenta, weil diese Beschreibung mit der von Kölliker (1847) gegebenen Schilderung über den Bau der Spermien von Ran a fusca nicht übereinstimmt!

In dem kurzen, dicken Mittelstück der Spermien von Rana esculenta fand Benda (1898) eine aus Mitochondrien gebildete Spiral bülle; eine Beobachtung, welche ich (Broman $1900: 2$ ) bestätigen konnte. Ich fand die betreffende Spiralhülle bei diesen Spermien besonders kurz, nur 2-3 Spiraltouren um die zentrale Mittelstückpartie (= Schwanzfaden und Centriolderivate) bildend. Nach Benda (1903) kann aber die Spirale auch etwas länger werden. Hinsichtlich des feineren Baues der analogen Hülle bei den Spermien von Rana fusca, scheint Benda (1903) nicht gern glauben $\mathrm{zu}$ wollen, dass eine Spirale hier in der Tat fehlen sollte. Dieser Autor scheint eher annehmen zu wollen, dass das Fehlen einer Spirale bei diesen Spermien "Symptom einer Degeneration oder unterbrochenen Reifung" des betreffenden Materials sein könnte.

Neue eigene Untersuchungen.

Der feinere Bau der Rana fusca-Spermien ist nicht gerade leicht $\mathrm{zu}$ eruieren, denn diese Spermien sind recht wenig resistent und werden daher in gewöhnlichen Trockenpräparaten und bei gewissen Färbungen (ohne vorhergehende Fixierung) sehr stark - und zwar im allgemeinen nicht besonders instruktiv - ver- 
ändert, ja unter Umständen sogar fast vollständig zerstört. Andererseits pflegen Ausstrichspräparate, welche ohne Eintrocknung fixiert und nachher gefärbt wurden, nur die Form der Spermien, nicht aber den wahren feineren Bau derselben gut hervorheben. - Nur durch das von mir früher (Broma n, 1900:1, p. 135) angegebene Gentianaviolett-Eintrocknungs-Verfahren ist es mir bisher gelungen, von reifen Spermien Präparate zu bekommen, welche die feinere Struktur der Spermien regelmässig zeigen.

\section{Bau der Spermien von Rana fusca.}

Die reifen Spermien von Rana fusca haben eine Totallänge von etwas mehr als $0,1 \mathrm{~mm}$. Der Kopf ist gewöhnlich etwa $45 \mu$, der Schwanz etwa $60 \mu$ lang. Die mit einer Körnchenhülle versehene, vordere Schwanzpartie, das Mittelstück oder Verbindungsstück, hat eine Länge von etwa $20 \mu$. - Die Dicke des Kopfes beträgt, wie schon P fl ü ge r (1883) richtig angegeben hat, etwa 1 ".

Dagegen ist Pflügers (1883), neulich von Gaupp (1904) zitierte, Angabe über die normale Kopflänge dieser Spermien $(51-60 \mu)$ unrichtig. Offenbar hat Pflüger (ebenso wie Leydig, 1877) die wahre hintere Kopfgrenze nicht gesehen, sondern die dickere Partie des Verbindungsstückes zu dem Kopfe mitgerechnet.

Wenn die Spermien in einer indifferenten Flüssigkeit (z. B. Humor aqueus aus den Augen desselben Tieres) lebend untersucht werden, findet man sie anfangs im allgemeinen ruhig liegend. Sie haben dann meistens die in Fig. 21 wiedergegebene Form.

Der Kopf ist in seiner vorderen Partie leicht seitwärts gebogen. Oft vermehrt sich diese Biegung relativ stark (wie in Fig. 22) oder betrifft dem ganzen Kopfe (wie in Fig. 24). Sowohl die vordere wie noch mehr die hintere Grenze des Verbindungsstückes sind nur mit grosser Schwierigkeit zu erkennen (vergl. Fig. 22); einen spiraligen Bau desselben konnte ich an lebenden Spermien nie unterscheiden. Bei diesen sind auch weder der Spiess, die Centriolkörner noch die in dem Verbindungsstück verlaufende Schwanzfadenpartie zu erkennen.

Wenn man in einem solchen Präparat (von Sperma mit Humor aqueus verdünnt) umhersucht, findet man bald einzelne Spermien, welche angefangen haben, sich $z u$ bewegen. Lebhafte, schlangenähnliche Bewegungen werden indessen nur von dem 
Verbindungsstück des Schwanzes ausgeführt. Die hintere nackte Schwanzfadenpartie führt nur wenig lebhafte und wohl schwächere Bewegungen (mit grösseren Wellen) aus. Der Kopf bewegt sich nur passiv entweder nach vorne oder um seine Achse rotierend oder beides.

Wenn zu einem solchen Präparat Wasser gesetzt wird, wird das früher relativ ruhige Präparat mit einmal wie durch einen Zauberschlag verïndert. Alle Spermien geraten dann in heftigste Bewegung. In den Cytoplasmaballen, welche teilweise frei im Präparate liegen (Fig. 23), teilweise an Spermien adhärieren (Fig. 22), fangen die eingeschlossenen Körnchen lebhafte, tanzende rotierende Bewegungen (sog. "B row n sche Molekularbewegungen“) an, welche unter Umständen Flimmersaumbewegungen simulieren können.

Die Spermienköpfe führen jetzt nicht nur passive sondern auch aktive Bewegungen aus, indem sie sich oft wellenförmig (in 2-4 langen Wellen) biegen (Fig. 19) und wieder strecken.

Die meisten Spermien scheinen indessen bald an Objektträger und Deckglas zu adhärieren ("Thigmotaxis“, Massart, 1888) und können dann also trotz lebhafter Bewegungen nicht mehr vorwärts kommen, wenn sie nicht durch äussere Eintlüsse wieder losgemacht werden.

Ich finde es wahrscheinlich, dass es besonders die vorderste Kopfpartie ist, welche diese Befestigung der Spermie vermittelt; denn wenn man ein eintrocknendes Ausstrichspräparat mit einem Glasstabende hin und her (im Sperma) streicht, bekommt man oft zehnfach in die Länge ausgezogene Spermienköpfe, deren vorderstes Ende fast unverindert ist und offenbar stärker als die übrige Partie der Spermie am Deckglas adhärent gewesen ist (vergl. Fig. 29).

Krankhaft veränderte oder abgestorbene Froschspermien sollten nach Massart (1888) keine Thigmotaxis mebr zeigen. Diese Angabe ist aber - glaube ich - so zu modifizieren, dass solche Spermien, wenn sie durch äussere Einflüsse losgemacht werden, sich nicht wieder am Deckglase aktiv befestigen können. Die vital eingetretene Adhäsion der Spermien wird nämlich beim Absterben derselben im allgemeinen nicht aufgehoben.

Wenn die Einwirkung des Wassers längere Zeit (z. B. 2-4 Stunden) gedauert hat, wird die von denselben ausgeübte Reiz- 
wirkung auf die Spermienköpfe offenbar noch grösser. Wenn diese nicht am Deckglase adbärent sind, rollen sie sich dann uhrfederăhnlich ein (Fig. 30) oder biegen sich ösenartig nach hinten um (Fig. 31) ${ }^{1}$ ).

Trotz dieser starken Veränderungen der Kopfform, welche - soviel ich bisher habe finden können - bis zum Absterben der Spermien unverändert persistieren, setzen die betreffenden Spermien noch lange fort, lebhaft umberzuschwimmen.

Diese krampfartigen Veränderungen der Kopfform finde ich in mehr als einer Beziehung interessant. Es liegt nämlich einerseits auf der Hand, dass so veränderte Spermien, obgleich sie noch mehrere Stunden umherschwimmen können, aller Wahrscheinlichkeit nach nicht mehr befruchtungsfähig sind. - Und andererseits finde ich es verdächtig, dass solche oder ähnliche Spermienformen zu unrichtigen Deutungen Anlass gegeben haben, und zwar, dass sie als normale Entwicklungsformen der Spermien betrachtet worden sind (vergl. z. B. die von v. la Valette St. George (1876) abgebildeten „Entwicklungsformen" der Bufo-Spermien! 1. c. Taf. XXXV, Fig. 57-60).

Wenn man ein mit Humor aqueus verdünntes Ausstrichspräparat ohne vorherige Eintrocknung oder Fixierung mit Gentianaviolett färbt, sterben die Spermien gleichzeitig mit der Färbung schnell ab. Sie färben sich zuerst fast gleichmässig und zwar ohne eine Formveränderung zu erleiden.

In solchen Präparaten kann man indessen bald konstatieren, dass die periphere Kopfpartie stärker als die zentrale gefärbt worden ist (Fig. 25a). Ob dieser Unterschied davon abhängt, dass die periphere Kopfpartie kompakter ist als die zentrale ist, oder nur davon, dass die zentrale Kopfpartie angefangen hat zu quellen, oder die Farbe wieder abzugeben, ist schwer abzumachen. Vielleicht wăre hier auch an einer beginnenden, durch chemische Prozesse veranlassten Entfärbung zu denken.

Wenn man nun recht viel Wasser zu dem Präparate zusetzt und dasselbe bei gewöhnlicher Zimmertemperatur eintrocknen lässt, quillt der Kopf im allgemeinen mehr oder weniger stark auf und verliert bald fast vollständig die Farbe. Nur in der

1) Schon Lee uwe nhoek (1687) hat dieses Phenomen beobachtet und dasselbe als ein Absterbungszeichen gedeutet (vergl. Textfig. 1). - Bei Rana esculenta beschreibt Ankermann (1857) ein ähnliches Phenomen. 
vordersten Kopfpartie (etwa dem vordersten Kopfzehntel) behält eine früher nicht unterscheidbare, kurze stabförmige Bildung die Farbe. Dieser Stab, welcher konstant an der einen Seite (und zwar an der konkaven) des Kopfes liegt, stellt den von mir früher beschriebenen Spiess (das Perforatorium) dieser Spermien dar.

Dieser Spiess kann schon 4-5 Stunden nach der Färbung, wenn das Präparat noch nicht vollständig eingetrocknet ist und die Spermienköpfe noch keine Quellung zeigen, sichtbar werden. $\mathrm{Zu}$ dieser Zeit pflegt dagegen das Verbindungsstück noch so stark und gleichmässig gefärbt zu sein, dass der feinere Bau desselben noch nicht $\mathrm{zu}$ erkennen ist.

Setzt man zu einem solchen Prăparat noch einmal Wasser zu und lässt dasselbe bis zum folgenden Tag in Zimmertemperatur eintrocknen, so findet man darin im allgemeinen eine grosse Menge Spermien, deren Verbindungsstück grösstenteils entfurbt worden ist. Die periphere Partie desselben bildet eine aus Körnchen bestehende Hülle, die von mir früher beschriebene Körnchenhülle, in deren Mitte sich der nackte Schwanzfaden als ein deutlich einfacher Faden bis zum Halsstücke (im Sinne Waldeyers, 1901) hinauf fortsetzt.

Unter Umständen ist die Körnchenhülle vollständig zerstört worden (Fig. 18). Man sieht dann deutlich, dass die früher von der Körnchenhülle umgebene Schwanzfadenpartie dieselbe Dicke wie der ursprünglich nackte Schwanzfadenteil besitzt und dass die Verbindung des Schwanzfadens mit dem Kopfe durch zwei miteinander eng verbundene, etwas ungleich grosse Körner (vergl. Fig. 18 u. 21) vermittelt wird. Diese Körner stellen die von mir (Broman, 1900:2, 1901: 1) früher beschriebenen Centriolderivate dieser Spermien dar und sind also als Halsstück im Sinne Waldeyers (1901) zu bezeichnen.

Das vordere, grössere Centriolkorn, welches, wie die Spermiogenese lehrt, in das hintere Kopfende hineingedrungen ist, stellt - wie ich früher angegeben hatte - den vergrösserten proximalen Centriol der jungen Spermatide dar. Das hintere kleinere Centriolkorn bildet den persistierenden Teil des distalen Spermatidencentriols (vergl. Fig. 1-21!). Offenbar sind die beiden Centriolkörner miteinander sehr stark verbunden; denn ich habe sie nie in einem Ausstrichspräparat getrennt gesehen. Die iso- 
lierten Schwänze (Fig. 17 u. 18) sind offenbar im allgemeinen durch ein Abbrechen des kaudalsten Kopfendes vom Kopfe getrennt worden.

Zwar treten bei der letzterwähnten Behandlung der Ausstrichspräparate nicht immer die Centriolderivate mit derselben Deutlichkeit wie z. B. der Spiess hervor: Ich habe aber jetzt in neuen Präparaten Tausende von sowohl unreifen wie (allem Anschein nach) reifen Spermien gesehen, deren Centriolderivate genau das von mir (1900:2) beschriebene Aussehen deutlich zeigen. Noch leichter ist es zu konstatieren, dass der Schwanzfaden - so wie ich (1900:2) ihn bescbrieben hatte - normaliter überall einfach ist und in der Mitte der Körnchenhülle verläuft (Fig. 16, 21 u. 26). Ich kann daher nicht umuin, die von Retzius (1906) gemachte indirelite Kritik meiner Befunde als vollständig verfehlt $\mathrm{zu}$ betrachten.

Teilweise auf meine neue Untersuchungen, vor allem aber auf meine alte spermiogenetische Untersuchungen gestüzt, kann ich die von Retzius (1906) in seinen Fig. 1 u. 7 (l. c. Taf. XXIII abgebildeten Ranaspermien mit Bestimmtheit als durch die Präparation hervorgerufene Kunstprodukte bezeichnen. Ähnliche Kunstprodukte habe auch ich - obgleich nur sehr selten - in einzelnen Praparaten gesehen. Die von Retzius als zwei Schwanzfäden aufgefassten beiden dunklen Streifen stellen nur den optischen Längsschnitt einer durch die Präparation etwas veränderten Körnchenhülle dar. In ganz ahnlicher Weise hat - wie ich (1900: 2) schon früher hervolgehoben habe - v. la Valette St. George (1886) die Spiralhülle der B u fo-Spermien irrig als Schwanzfadenpartien aufgefasst (l.c.Taf.XIV, Fig. 3, 4 u. s).

Die vorderste Partie der zu einem kompakten Zylinder umgewandelten Körnchenhülle hatte sich wohl in den erwähnten, von Retzius abgebildeten Spermien etwas stärker als die übrige Partie derselben gefärbt und imponierte daher als zwei nebeneinander gelegene Körner, welche von Retzius als Centriolderivate aufgefasst wurden. Bis $\mathrm{zu}$ dem in Fig. 13 abgebildeten Entwicklungsstadium ${ }^{1}$ ) nehmen indessen die wahren Centriol-

1) Von demselben Entwicklungstadium batte ich neulich Gelegenheit Herrn Professor Retzius einige Spermatiden zu demonstrieren. Zu erwähnen ist, dass Professor Retzius mich dann dringend aufforderte, meine Untersuchungen über die Histogenese der Rana-Spermien sofort zu veröffentlichen. 
derivate eine ganz andere Lage ein. Durch Untersuchung von älteren unreifen Spermien in Ausstrichspräparaten habe ich die Möglichkeit anschliessen können, dass der proximale Centriol in den allerspätesten Entwicklungsperioden in kleineren Körnern geteilt werden sollte.

Betreffs des feineren Baues des Schwanzes möchte ich hier noch folgendes bemerken.

Der im Leben einfache Schwanzfaden ist aller Wahrscheinlichkeit nach von dünneren Fibrillen zusammengesetzt (in ähnlicher Weise wie besonders Ballowit $\mathrm{z}$ bei so vielen anderen Spermien festgestellt hat); bei gewisser Behandlung habe ich nämlich einen Zerfall des Schwanzfadens in zwei Fibrillen beobachten können (Fig. 17 u. 25).

Die Körnchenhülle kann bei gewisser Behandlung ein spiraliges Aussehen annehmen (Fig. 17). Da ich indessen dieses nur relativ selten mit Sicherheit habe beobachten können, möchte ich, bis stärkere Beweise dafür vorgebracht werden können, dass die Körnchenhülle als Kunstprodukt aus einer Spiralliülle hervorgegangen sei, eher das Umgekehrte annehmen.

Wenn man die Spermien von Rana fusca, ohne sie eintrocknen zu lassen, in der Zenkerschen Flüssigkeit fixiert und mit Eisenhämatoxylin nach $\mathrm{M}$. Heidenhain fürbt, findet man unter Umständen, wenn die Entfärbung recht lange gedauert hat, dass die hinterste Kopfpartie die Farbe behalten hat, obgleich die übrigen Partien des Kopfes fast vollständig entfärbt worden sind (Fig. 24). Im allgemeinen geht die gefärbte Kopfpartie in die ungefärbte allmăhlich über. Bisweilen findet man aber zwischen den dunklen und hellen Kopfpartien eine recht scharfe Grenze. In diesen Fällen zeigt die hintere dunkle Kopfpartie eine frappante Ähnlichkeit mit dem grossen proximalen Centriolderivat der Salamanderspermien (vergl. Meves, 1897). Indessen zeigt die Spermiogenese, dass es sich hier um etwas ganz anderes handeln muss. Vielleicht wird hier der Farbenunterschied zwischen den verschiedenen Kopfpartien nur durch eine etwas verschiedene chemische Zusammensetzung des Chromatins hervorgerufen. Denn ein ahnlicher Unterschied tritt bei Färbung mit Gentianaviolett am hinteren Kopfende nicht hervor; was man wohl hätte erwarten können, wenn verschiedene physikalische 
Eigenschaften diese verschiedene Farrbbarkeit mit Eisenhämatoxylin veranlasst bätten.

Eine gewissermassen ähnliche verschiedene Färbbarkeit tritt dagegen bei Färbung mit Gentianaviolett im vorderen Kopfteil oft hervor, wenn die Spermien nicht fixiert und vor der Farbung eingetrocknet waren. Die vorderste Kopfpartie quillt dann oft nicht anf, während die hintere grössere Kopfpartie fast regelmässig einer starken Quellung unterliegt. Hierbei wird diese Kopfpartie nicht nur dicker, sondern gewöhnlich auch länger als früher.

Dass die verschiedene Färbbarkeit der betreffenden Kopfpartien in diesem Falle wenigstens teilweise von verschiedenen physikalischen Eigenschaften abhängt, unterliegt wohl keinem Zweifel. Die vorderste Kopfpartie ist kompakter geblieben und schliesst also die färbbare Substanz mehr konzentriert in sich ein.

Warum ist aber diese vorderste Kopfpartie kompakter geblieben? Aller Wahrscheinlichkeit nach, weil diese Kopfpartie schon, wenn die Spermien noch lebend waren, am Deckgläschen so fest adhärierte, dass diese Adhäsion für eine Quellung dieser Kopfpartie ein mechanisches Hindernis darstellte. Die hintere, grössere Kopfpartie, welche am Deckgläschen nicht im Leben adhärent wird, ist dagegen - wenn sie beim Eintrocknen zu quellen tendiert - unverhindert, sich (sowohl seitwärts wie im allgemeinen auch nach hinten) nach Belieben auszubreiten.

Selbstverständlich führt auch das Eintrocknen zu einer starken Adhăsion der eintrocknenden Spermienpartien. Wenn nun beim Eintrocknen zufalligerweise das Schwanzende zuerst betroffen wird, so, dass es am Deckgläschen befestigt wird ehe der Kopf zu quellen angefangen hat, muss der letztgenannte sich wellenförmig (vergl. Fig. 26) oder in anderer Weise biegen, um bei der Quellung genügend Raum zu finden. Die Form solcher Kunstprodukte (Fig. 26) erinnert oft stark an die normale Form der Pelobates-Spermien.

Nicht nur in gewöhnlichen Trockenpräparaten, sondern auch in solchen Fällen, wenn man die Spermien für gewisse schädliche Reagentien (z. B. Hămatoxylin ohne vorherige Fixierung oder kurze Mazeration in Wasser) aussetzt, findet man, dass die vorderste Kopfpartie viel resistenter als der übrige Kopfteil ist. Bei Färbung mit Hämatoxylin ohne vorhergehende Fixierung 
zerfăllt die grösste Partie des Kopfes bald in Körnchen (Fig. 27), während die vorderste Kopfpartie fast unverăndert bleibt. Die betreffenden Körnchen können fortgeschwemmt (vielleicht auch aufgelöst) werden. So erklärt es sich, dass man in solchen Präparaten oft ganz isolierte Kopfspitzen (Fig. 28) findet.

Ich finde es nicht unwahrscheinlich, dass diese Kopfspitzen (Fig. 27 und 28), welche mit den wahren Spiessen oder Perforatorien dieser Spermien (vergl. Fig. 19-21) nicht zu verwechseln sind, nur durch ihre Adhăsion am Deckgläschen von einem partiellen Untergang gerettet worden sind. Denn bei anderer Behandlung treten Bilder hervor, welche es glaubhaft machen, dass nur das seitlich liegende Perforatorium eine an und für sich sehr resistente Kopfpartie darstellt, und dass der übrige Teil der vorderen Kopfspitze von etwa derselben Konsistenz wie der grössere Kopfteil ist (vergl. z. B. Fig. 20).

Sehr bemerkenswert finde ich es, dass die lebenden Spermien sich in der Weise an das Deckgläschen befestigen, dass das Perforatorium immer in Profil zu liegen kommt (vergl. Fig. $16,19,20 \mathrm{a}, 21$ und $25 \mathrm{~b}$ ). So viel ich bisher habe finden können, sind Enface-Lagen des Perforatoriums (Fig. 20 b) nur in Trockenprăparaten von solchen Spermien zu beobachten, welche unmittelbar vor dem Eintrocknen in der Flüssigkeit ganz frei (d. h. ohne Adhäsion an das Deckglasschen) lagen. - Vielleicht hăngt diese konstante Profillage der lebend adhärenten Spermien nur von der oben erwahnten Kopf biegung ab, welche einerseits natürlich in dem engen Raum zwischen Objektträger und Deckgläschen zu einer Profillage einladen muss, und andererseits wohl für eine Adhäsion der Kopfspitze in Enface-Lage direkt hinderlich ist.

\section{Bau der Spermien von Rana esculenta, Rana arvalis und Rana mugiens.}

Obgleich ich es nunmehr als etwas unvorsichtig betrachte, den feineren Bau von reifen Spermien zu beschreiben, ehe man die Entwicklung derselben genau verfolgt hat, gebe ich hier zur Komparation Abbildungen der Spermien von noch drei Rana-Arten.

Die Spermien von Rana esculenta (Fig. 32-34) nehmen - gleich wie diejenigen von Rana fusca - bei verschiedener Behandlung ein recht verschiedenes Aussehen an. Dieses gilt 
besonders von dem Verbindungsstück. Unter Ümständen nimmt dasselbe das in Fig. 33 abgebildete Aussehen an, welches die Existenz einer vorderen Verzweigung des Schwanzfadens simuliert. Dieses Bild ist indessen mit Sicherheit als ein Kunstprodukt zu betrachten, welches durch den optischen Längsschnitt einer alterierten Spiralhülle hervorgerufen wird.

Diese Spermien besitzen nämlich, wie schon Benda (1898) beschrieben hat, eine Spiralhülle in dem kurzen Verbindungsstück. Diese Spiralhülle umgiebt die von mir (1900:2) beschriebenen Körner, welche die Verbindung des einfachen Schwanzfadens mit dem Kopfe vermitteln (Fig. 32). Da diese Körner dasselbe Aussehen wie die Centriolderivate anderer Anurenspermien (Ran a fusca and Bombinator igneus) zeigen und bei derselben Behandlung hervortreten, sind sie aller Wahrscheinlichkeit nach auch hier als Centriolderivate $\mathrm{zu}$ betrachten. $\mathrm{Ob}$ sie die fast unveränderten Centriolen der jungen Spermatide (wie bei Bombinator) darstellen, oder ob sie nur teilweise die ursprünglichen Spermatidencentriolen (wie bei $R$ a n a f us ca) repräsentieren, müssen spermiogenetische Untersuchungen lehren.

Der von v. la Valette St. George (1886) entdeckte kleine knopfförmige Spiess dieser Spermien ist im allgemeinen leicht zu erkennen. - Der im Leben einfach aussehende Schwanzfaden ist wahrscheinlich aus Fibrillen zusammengesetzt, denn ich habe bisweilen Andeutungen $\mathrm{zu}$ einem Zerfallen in feineren Fäden (Fig. 33) gesehen.

Die Spermien von Rana arvalis (Fig. 35) sind denen von Rana esculenta wie bekannt sehr ahnlich. Nur habe ich hier im allgemeinen eine etwas längere Spiralhülle, einen kürzeren Schwanzfaden und einen vorn etwas spitzeren Kopf gesehen. Da ich indessen Spermien von nur einem einzigen Exemplar bisher gesehen habe, kann ich aber nicht ganz sicher sein, dass diese Unterschiede konstant zu finden sind.

Die Spermien von Rana mugiens (Fig. 36 und 37) sind ebenfalls denen von Rana esculenta sehr ähnlich. Wie ich schon früher hervorgehoben habe (B rom a n, 1900:2), ist aber hier das Perforatorium etwas mehr ausgebreitet. Es sitzt als eine sehr kleine Mütze am vorderen Kopfende (Fig. 37). - Die Spiralhïlle fand ich bei diesen Spermien ganz besonders deutlich und zwar auch bei lebenden Spermien. 
Die Spermien der letzterwähnten drei Rana-Arten sind also einander so ähnlich, dass es berechtigt sein kann, ihre Form als gewöhnlicher Typus der bisher untersuchten Rana-Spermien aufzustellen.

Von dieser Form weicht aber diejenige der Spermien von $R$ an a fusca so beträchtlich $a b$, dass ich es für angemessen finde, sie als einen besonderen Typus der Ranaspermien aufzustellen. Soviel wir bis jetzt wissen, hat ausser Rana fusca nur Rana agilis (de l'Isle, 1873) Spermien von diesem Typus.

\section{Ueber die Entwicklung der Spermien von Rana fusca.}

Frühere Untersuchungen.

Aus älterer Zeit liegen mehrere Untersuchungen über die Histogenese der Spermien von Rana fusca vor. Kölliker (1847), Remak (1854), Ankermann (1857), Neumann (1868, 1875), von la Valette St. George (1871 und 1876) und Gruenhagen (1885) haben dieses Thema mehr oder weniger eingehend behandelt. Da indessen den betreffenden Autoren im allgemeinen nur sehr unvollkommene histologische Arbeitsmethoden zur Verfügung standen, darf es nicht Wunder nehmen, dass die Ergebnisse dieser Untersuchungen auch sehr unvollkommen wurden. So viel wurde indessen von v. la Valette St. George festgestellt, dass der Spermienkopf aus dem Spermatidenkern, der Spermienschwanz aus dem Spermatidenzytoplasma hervorgeht.

In neuerer Zeit ist dieses Thema eingehender nur von Bertacchini $(1891,1896)$ behandelt worden. Dieser Autor konnte aber - so viel ich verstehe - unsere Kenntnisse über die Histogenese der Ranaspermien nicht nennenswert durch Beobachtungen von bestehendem Wert vergrössern.

Nach der Einführung der Hämatoxylin-Eisenalaunfärbung von M. Heidenhain (1892, 1894, 1896), - welche Methode bekanntlich ein genaueres Studium u. a. auch von den Centriolen und ihren Derivaten ermöglicht, und daher in der spermiogenetischen Forschung von epochemachender Bedeutung gewesen ist, haben - so viel ich weiss - nur Benda (1898) und ich (Broman, 1900, 1901: 1) Mitteilungen über die Spermjogenese bei Rana veröffentlicht. Unsere Angaben hierüber waren indessen 
nur anmerkungsweise in Publikationen anderen Inhalts eingefügt und daher sehr unvollständig.

Benda (1898) sagt hierüber nur folgendes: "Von den Amphibien zeigen Anuren (Rana, Bombinator) nur geringfügige Verwendung der Centrosomen, die fast in ihrer ursprünglichen Lage und Form verbleiben, so dass das zentrosomale Mittelstück nur kornartig bleibt."

Selbst habe ich die Entwicklung der Spermatidencentriolen bei Rana fusca nur in der obenerwăhnten, schwedischen Arbeit (1900:2) kurz beschrieben und durch acht Textfiguren (den Fig. 1, 2, 4, 5, 7-11 in dieser Arbeit entsprechend) in einer anderen Arbeit (Broman 1901: 1, p. 127) illustriert.

\section{Eigene Untersuchungen.}

Die Spermatiden von $R$ a $n$ a fusca sind relativ kleine Zellen. Sie sind ein Drittel kleiner als die Spermatiden von Bombinator und kaum halb so gross wie diejenigen von Salamandra. Die Kerne sind auch entsprechend kleiner. Dagegen haben die Zentralkörper oder Centriolen der jungen Spermatiden bei Rana etwa dieselbe Grösse wie diejenigen der jungen Bombinator- und Salamandraspermatiden.

In der Entwicklung der Ranaspermatiden unterscheide ich drei Perioden. Wahrend der ersten Entwicklungsperiode entsteht der Schwanzfaden, und der Kern der Spermatide setzt sich mit den Centriolen in Verbindung (Fig. 1-6). Die $z$ weite Entwicklungsperiode ist durch gewisse Umwandlungen der Centriolen und durch Umlagerung des Cytoplasmas und der Mitochondrien charakterisiert (Fig. 7-11). Während der dritten Entwicklungsperiode werden die überflüssigen Cytoplasmapartien abgeschnürt und die Spermienköpfe nehmen ihre charakteristische, stark in die Lange ausgezogene Form an (Fig. 12, 13, 22).

\section{Erste Fntwicklungsperiode.}

Bei den jüngsten von mir beobachteten Ranaspermatiden waren die Centriolen als paarige Körnchen unmittelbar unter der Zellwand $\mathrm{zu}$ erkennen. Ihre Verbindungslinie lag senkrecht zur Zellwand (vgl. Fig. 1, Taf. XIX). Von dem distalen Centriol war schon ein Schwanzfaden ausgewachsen. Der proximale Centriol war noch durch eine dicke Cytoplasmapartie von dem zentral gelegenen, sphärischen Kern getrennt. Etwas seitwärts von den 
Centriolen war im Cytoplasma ein homogener Körper zu erkennen, welcher wahrscheinlich als ein Idiozom aufzufassen ist.

In dem nächstfolgenden Stadium schiebt der. Kern den Centriolen einen Fortsatz entgegen (Fig. 2), welcher bald mit dem proximalen Centriol in Verbindung tritt (Fig. 3 und 4).

Diesen Fortsatz, welchen ich als den sichtbaren Ausdruck einer von den Centriolen ausgehenden Reizwirkung (Mikrocentrotaxis) auf den Kern betrachte, habe ich (Broman, 1901: 1) Empfangniszapfchen benant und dem Empfängnishügel der Eier gewissermaßen parallel gestellt.

Das Empfängniszåpfchen des Spermatidenkerns wird in den folgenden Stadien (Fig. 5 und 6) wieder vollständig eingezogen, und Hand in Hand hiermit werden auch die an demselben fixierten Centriolen und die proximale Partie des Schwanzfadens in das Innere des Zellkörpers aufgenommen.

$\mathrm{Zu}$ bemerken ist indessen, dass die an dem distalen Centriol fixierte Zellwandpartie gleichzeitig miteingezogen wird, was wenigstens wenn die Spermatiden eng zusammenliegen - zu der Ausbildung eines engen Kanals (vgl. Fig. 5 und 6) führt, welcher die proximale Schwanzfadenpartie in sich birgt. Die Wände dieses Kanals werden von der ursprünglichen Zellwand gebildet. Streng genommen liegt also die betreffende Schwanzfadenpartie noch vollständig extracellulär (Fig. 6). Bald obliteriert aber der betreffende Kanal, und die proximale Schwanzfadenpartie wird dann intracellular.

\section{Zweite Entwicklungsperiode.}

Die Centriolen, welche in der ersten Entwicklungsperiode keine Formveränderungen erlitten hatten, fangen jetzt an, sich umzuformen. Der proximale Centriol dringt in den Kern hinein (Fig. 7) und vergrössert sich in allen Richtungen, bis er etwa doppelt grösser als früher wird. Der distale Centriol, welcher ausserbalb der Kernmembran liegen bleibt, wird ebenfalls grösser, vergrössert sich aber nicht gleichmăssig, sondern nimmt die Form einer gegen dem Schwanzfaden quer gestellten, kreisrunden Platte (Fig. 7) an. In einem nächstfolgenden Stadium zerfällt diese Centriolplatte in eine periphere ringförmige Partie (Fig. 8) und ein zentral gelegenes Körnchen. Dieses Körnchen, welches die Verbindung des Schwanzfadens mit dem proximalen Centriol - 
und hierdurch auch mit dem Kopfe - vermittelt, ist in den Figuren 8-10 unter dem stark gefärbten Centriolring versteckt. ${ }^{1}$ ) In dem Stadium Fig. 11 verschwindet aber dieser Ring in loco, indem er seine Farbbarkeit verliert (und wohl in dem Cytoplasma aufgelöst wird) und gleichzeitig tritt das zentrale Körnchen des distalen Centriols deutlich hervor.

Dieses Centriolkörnchen ist jetzt etwas grösser, als der ganze distale Centriol der Spermatide ursprünglich war (vgl. Fig. 11 und 1); er ist dagegen deutlich kleiner als der angewachsene proximale Centriol (siehe Fig. 11).

Nach dem Verschwinden des Centriolringes verändern die Centriolderivate der Spermatide weder Form noch Lage, sondern sind noch bei der reifen Spermie als zwei ungleich grosse Körnchen wiederzufinden, deren Verbindungsiinie in der Lăngsachse der Spermie und ihr parallel liegt (vgl. Fig. 11-13 u. 16).

Anfang dieser zweiten Entwicklungsperiode werden - bei der hier benutzten Prăparationsmethode - unmittelbar kaudalwärts vom distalen Centriol eine grosse Menge feinster Körnchen (Fig. 7) sichtbar, welche ein relativ grosses, von der proximalsten Schwanzfadenpartie durchgesetztes Aggregat bilden. Nach dem späteren Schicksal dieser Körnchen zu urteilen, sind sie mit den Mitochondrien Bendas identisch.

Der Körnchenhaufen dehnt sich in den folgenden Stadien allmäblich nach binten aus und bildet zuletzt eine kaudalwärts sich allmählich verjüngende Körnchenhülle um der intrazellularen Schwanzfadenpartie umher (vergl. Fig. 7-11).

Gleichzeitig hiermit verschiebt sich auch die ganze Cytoplasmamasse der Spermatide kaudalwarts. Der zu dem Spermienkopf sich umformende Spermatidenkern wird hierbei allmählich seiner Cytoplasmahülle fast vollständig beraubt. Ende der zweiten Entwickelungsperiode (Fig. 11) ist diese Hülle im allgemeinen nicht mehr deutlich zu erkennen.

Was die Kopfanlage auf diese Weise an Cytoplasmabekleidung verliert, gewinnt also die Schwanzanlage, deren intrazelluläre Partie hierbei beträchtlich länger wird. Für die Länge der obenerwähnten Körnchenhülle des Schwanzes hat diese Cytoplasmaverschiebung grosse Bedentung. Denn ebensoweit, wie die

1) In Fig. 14 ist der Centriolring distociert worden und das betreffende Körnchen daher gleichzeitig mit dem Centriolring sichtbar. 
Cytoplasmahülle des Schwanzes kaudalwärts vordringt, dehnt sich auch die Körnchenhülle desselben aus (vergl. Fig. 9-11). Diese Hülle hat jetzt - (Ende der zweiten Entwicklungsperiode) schon zwei Drittel ihrer definitiven Länge erreicht. Die Länge der Kopfananlage beträgt zu dieser Zeit nur etwa ein Viertel der definitiven Kopflänge.

Schon in der ersten Entwicklungsperiode (vergl. Fig. 4 u. ̃) tritt am Spermatidenkern - und zwar etwas seitlich vom vorderen Pole - ein Bläschen auf, welches - nach unseren Erfahrungen bei verwandten Tieren zu urteilen - aller Wahrscheinlichkeit nach als ein Idiozomderivat aufzufassen ist.

Auch in der zweiten Entwicklungsperiode bleibt dieses Bläschen etwas seitlich liegen (vergl. Fig. 7-11). Bei der Verlängerung der Spermienkopfanlage plattet sich dasselbe $a b$ und verlängert sich ein wenig in der Längsrichtung des Kopfes. Die an der einen Seite des vorderen Kopfendes fixierte Bläschenwand verdickt sich zuletzt (Fig. 11), während die laterale Bläschenwand dünn bleibt. Gleichzeitig verkleinert sich das Bläschenlumen immermehr, bis es zuletzt vollständig obliteriert (vergl. Fig. 11 und 12). Wir erkennen jetzt in dem betreffenden Bläschen die Anlage des Perforatoriums oder des Spiesses.

Wie wir sehen, sind also schon Ende der zweiten Entwicklungsperiode, welche als die wichtigste Formbildungsperiode $z u$ bezeichnen ist, die Spermatiden in Spermien umgewandelt, welche sich von reifen Spermien histologisch hauptsächlich nur dadurch unterscheiden, dass der Kopf kürzer und dicker und die Cytoplasmahülle des Schwanzes voltıminöser ist.

Man könnte daher die

\section{dritte Entwickelungsperiode}

auch die Wachstums- und Reifungsperiode benennen.

Schon Anfang dieser Periode (Fig. 12) beginnen die überHiüssigen Cytoplasmapartien sich in Form kleiner Ballen von den Spermien abzuschnüren. Die Cytoplasmahülle des Schwanzes wird hierbei bald so stark reduziert, dass sie im allgemeinen nicht mehr als eine besondere Hülle nach aussen von der Körnchenhülle deutlich zu erkennen ist. - Die allmählich degenerierenden Cytoplasmaballen sind noch längere Zeit zwischen den kaudalen Partien der Spermienschwänze zu sehen, an welchen sie in Hodenausstrichspräparaten oft adhärieren (Fig. 22). 
Wie schon oben angedeutet, verlangert sich wăhrend dieser Periode die Körnchenhülle, so dass sie bei reifen Spermien etwa ein Drittel lănger als in dem ersten Stadium dieser Periode wird. Ob gleichzeitig hiermit auch die von dieser Hülle eingeschlossene Schwanzfadenpartie entsprechend viel in die Länge wächst, oder ob die Körnchenhülle sich nur kaudalwärts an einem Schwanzfaden verschiebt, welcher schon früher seine definitive Länge erreicht hat, lässt sich natürlich an Schnittpräparaten nicht feststellen. (Sicher ist dagegen, dass der Schwanzfaden wăhrend dieser Entwickelungsperiode etwas dicker wird.)

Bemerkenswert ist übrigens, dass die obenerwähnten Körnchen - soweit ich bisher habe feststellen können - gewöhnlich keine deutlich spiralige Gruppierung annehmen.

Die Centriolderivate verändern sich - wie schon erwähnt während dieser Periode nicht nennenswert (vergl. Fig. 12, 13, 16, 18 und 21).

Wie schon oben angedeutet wurde, verlängert sich der Spermienkopf wăhrend dieser Periode so stark, dass er am Ende der Periode etwa viermal länger als am Anfang derselben ist. Diese Verlängerung findet wohl hauptsächlich durch Wachstum statt. In der ersten Hälfte dieser Periode handelt es sich aber wahrscheinlich auch um eine Umformung, denn der Spermienkopf wird zu dieser Zeit deutlich dünner (vergl. Fig. 11-13). Das Chromatin konzentriert sich indessen gleichzeitig und nimmt ein mehr homogenes Aussehen an; was allein die Verdünnung des Kopfes erklären könnte.

Bei dieser starken Verlängerung des Spermienkopfes wird das vordere Ende desselben spitz ausgezogen, und Hand in Hand hiermit wird auch der hier einseitig fixierte Spiess entsprechend länger und spitzer. Diejenige Kopfseite, welche den Spiess trägt, bleibt indessen oft in der betreffenden Höhe oft ein wenig kürzer als die entgegengesetzte Seite, was zu einer schwachen Biegung des vorderen Kopfendes Veranlassung gibt (vergl. Fig. 13 u. 21).

Nicht ganz reife Spermien zeigen oft nach dem obenerwähnten Gentiana-Eintrocknungs-Verfahren einen stark gefärbten Spiess, welcher sich gegen das vordere Kopfende hin nicht wie bei den reifen Spermien (Fïg. 21) allmählich verjüngt, sondern etwa an der Mitte eine mehr oder weniger hohe knopfförmige Anschwellung trägt (Fig. 16). Da ich diese Anschwellung bei 
frischen Spermien desselben Stadiums nie gesehen habe, nehme ich an, dass sie durch eine bei dieser Behandlung aufgetretene postmortale Veränderung der Idiozomblase (deren laterale Wand sich vielleicht noch nicht so fest wie später an die mediale Blasenwand fixiert hat) zu Stande gekommen ist.

Wenn wir die oben geschilderte Histogenese der Ranaspermien mit derjenigen verwandter Spermienformen vergleichen, so finden wir sie im grossen gesehen miteinander sehr übereinstimmend.

In einem Punkte weicht indessen die Spermiogenese bei Rana fusca von denjenigen aller in dieser Hinsicht bisher untersuchten Tieren ab, deren Spermatiden einen im Zellinnern entstandenen Centriolring besitzen: Der aus dem distalen Centriol stammenden Centriolring verschwindet nämlich hier in loco, also ohne (ganz oder teilweise) im Voraus eine Kaudalwärtswanderung ausgeführt zu haben (vergl. Fig. 10-12).

Vielleicht ist die $\mathrm{nachste}$ Ursache hierzu darin zu suchen, dass die Mitochondrien sich relativ frühzeitig und zwar ehe der Ring gebildet worden ist, um die intrazellulare Schwanzfadenpartie umher gruppieren (Fig. 7) und so wohl ein mechanisches Hindernis für die Kaudalwärtsverschiebung des Ringes bilden.

Indessen erscheint die Bedeutung des Centriolringes in der Spermiogenese nach dieser Beobachtung - wenn möglich noch rätselhafter als früher. Venn die Annahme z. B., er habe die Aufgabe, die Cytoplasmahülle der Spermie kaudalwärts abzugrenzen oder die Kaudalwärtsverschiebung dieser Hülle zu leiten, wird hier hinfällig.

Einfacher wäre es anzunehmen, dass der Ring (wenigstens in gewissen Fallen) eine Centriolpartie darstellt, welche nur durch übermässiges Wachstum entstanden ist und daher als für die spätere Funktion unnötig oder schädlich abgeworfen wird. Wir würden dann auch eine einfache Erklärung für die Tatsache gefunden haben, dass der distale Centriol der Bombinatorspermatide, welcher sich nicht nennenswert vergrössert, auch keinen Ring bildet (B roman, 1900: 1). Weniger befriedigend wird indessen diese Annahme, wenn man sie auf solche Tiere transponiert, deren distaler Spermatidencentriol ganz und gar in einen Ring umgebildet wird. 
Von den übrigen Ergebnissen meiner Spermiogenetischen Untersuchung finde ich besonders folgende bemerkenswert:

1. Dass die an der Peripherie der jungen Spermatide liegenden Centriolen ${ }^{1}$ ) nicht aktiv (d. h. unter Vermittlung von Schwanzfaden-Bewegungen) auf einen unveränderten Kern zu einwandern, sondern durch einen Kernfortsatz, das Empfängn is zäp f chen, von der Zellenperipherie abgeholt werden (vergl. Fig. 1-6).

Eine ähnliche Beobachtung hatte Meves (1898) schon früher bei der Ratte gemacht. Andeutungsweise ist ein solches Empfängniszäpfchen nach Meves (1899) auch bei den Spermatiden des Meerschweinchens $\mathrm{zu}$ finden. Hier spielt jedoch die aktive Centriolwanderung für die Verbindung der Centriolen mit dem Kerne die Hauptrolle ( $\mathrm{M}$ eve s).

2. Dass die proximale Schwanzfadenpartie der Spermatide zunächst in einem engen Zellwandkanal zu liegen kommt (vergl. Fig. 5 u. 6).

Eine ähnliche Beobachtung hat zuerst Meves (1897) bei normalen Salamanderspermatiden gemacht. Bei Tritonspermatiden fand Benda (1898) dieselbe Erscheinung besonders deutlich ausgesprochen; und bei zweischwänzigen Riesenspermatiden von S a lamander konnte ich (B rom an, 1902) die Existenz eines die beiden Schwanzfäden gemeinsam einschliessenden Zellwandkanals (vgl. Taf. $47-48$, Fig. 49 und 51) feststellen.

3. Dass das Idiozombläschen einseitig an dem vorderen Kopfende liegen bleibt (Fig. 7-11), so dass das Perforatorium der reifen Spermie wie ein Fingernagel dem Kopfe anzusitzen kommt (Fig. 21 und 25 b).

Eine ähnliche seitliche Anlagerung des Perforatoriums ist von Ballowitz (1890) and mir (1900:2) bei Triton taeniatus und von Retzius (1906) bei Pleurodeles Waltlii beobachtet worden.

4. Dass die Mitochondrien des Spermienschwanzes wenigstens so viel meine bisherigen Hodenschnit tpräparate zeigen - zu einem Spiralfaden nicht verschmelzen.

Ich finde dieses bemerkenswert, weil bei den übrigen in dieser Hinsicht genauer untersuchten Anurenspermien - mit Ausnahme von den Pelobatesspermien (Broman, 1901: 2), denen eine Mitochondrienhülle vollständig fehlt - immer die Existenz einer mehr oder weniger deutlichen Spiralhülle zu konstatieren war (Benda, 1898, 1903, B roman, 1900: 2). - Indessen betrachte

1) $=$ Zentralkörper. 
ich diese Frage als noch nicht mit absoluter Sicherheit beantwortet. Denn wie oben erwähnt, habe ich bei gewisser Behandlung der Ausstrichspräparate einzelne Spermien von Rana fusca gesehen, deren Verbindungsstück ein spiraliges Aussehen erkennen liess, und ich finde es nicht ganz unmöglich, dass verbesserte Präparationsmethoden auch bei den reifen Ranafuscaspermien eine Spiralhülle als normale Bildung enthüllen werden.

5. Dass schon im Stadium Fig. 12 das überflüssige Cytoplasma von der jungen Spermie abgeschnürt wird.

Ein ähnlicher Abschnürungsprozess ist bei vielen anderen $\mathrm{Ob}$ jekten beobachtet worden (z. B. bei Meerschweinchen von Me ves [1899] und bei Bombinator von mir [1900: 1]).

6. Dass bei gewisser Behandlung der.unreifen Spermien am Perforatorium eine knopfförmige Anschwellung (Fig. 16) auftritt, welche als Präparations - Kunstprodukt aufzufassen ist.

Von den Ergebnissen meiner Untersuchungen von reifen Spermien hebe ich besonders hervor:

1. Dass die Spermien von Rana fusca ein stabförmiges Perforatorium besitzen, welches konstant an der einen (und zwar an der konkaven) Seite des Kopfes liegt (Fig. 21).

2. Dass der ganze Kopf im übrigen wahrscheinlich physikalisch gleichmässig gebaut ist (Fig. 16 und 20).

3. Dass aber bei gewisser Behandlung die vorderste Kopfpartie resistenter als die übrige erscheint (Fig. 26-29).

4. Dass es indessen wahrscheinlich nur die wahrend des Lebens stattgefundene Adhäsion dieser Kopfpartie an das Deckgläschen ist, welche die an der Seite des Perforatoriums liegende Kopfpartie vor Quellung und Zellfall schützt.

5. Dass diese vitale Adhasion des vordersten Kopfteils, welche beim Absterben der Spermie im allgemeinen nicht aufgehoben wird, regelmässig so stattfindet, dass das Perforatorium Profillage einzunehmen kommt.

6. Dass die hinterste Kopfpartie nach Fixierung in der Z enkerschen Flüssigkeit und Farbung mit Eisenhämatoxylin die Farbe länger als die übrige Kopfpartie behält (Fig. 24), was wohl an einer chemișchen Verschiedenbeit hindeutet. 
7. Dass die von $P f l u ̈ g$ er (1883) angegebene (von G a u p p 1904 zitierte) Zahl über die normale Länge des Kopfes der Rana-fusca-Spermien unrichtig ist.

8. Dass die Ursache zu dieser unrichtigen Angabe vielleicht darin $\mathrm{zu}$ suchen sein könnte, dass die Spermienköpfe beim Eintrocknen im allgemeinen nicht nur dicker; sondern auch etwas länger werden; dass sie aber am wabrscheinlichsten darin liegt, dass $P$ f $l$ ü g e $r$ nicht nur den wahren Kopf sondern auch die dickere Partie des Verbindungsstückes als zum Kopfe gehörend gemessen hat.

9. Das das Halsst ü ck der Rana-fusca-Spermien aus zwei eng verbundenen, etwa ungleich grossen Körnern besteht, von welchen das vordere, grössere aus dem ganzen proximalen Spermatidencentriol hervorgegangen ist, wahrend das hintere nur aus der mittleren Partie des distalen Spermatidencentriols herstammt.

10. Dass das vordere Centriolkorn innerhalb der inneren ${ }^{1}$ ) Kopfmembran (= der früheren Kernmembran) liegt.

11. Dass bei den Rana-fusca-Spermien keine Centriolkörner existieren, deren Verbindungslinie senkreckt gegen die Spermiumachse liegt (entgegen Retzius, 1906).

12. Dass der von dem hinteren Centriolkorn ausgehende Schwanzfaden, wenn er nicht durch besondere Behandlung zum Zerfallen gebracht worden ist (Fig. 17, 25 und 33), einfach ist und in der Mitte des Verbindungsstückes verläuft (Fig. 16-18, 21 u. 26.) (Entgegen Retzius, 1906).

13. Dass die von Retzius (1906) im Gebiete des Verbindungsstückes abgebildeten beiden „Schwanzfäden “ aller Wahrscheinlichkeit nach nur der optische Längsschnitt einer durch die Präparation veränderten Körnchenhülle und mit Sicherheit keine wahren Schwanzfäden darstellen.

14. Dass der Schwanzfaden der Ranaspermien überall dieselbe Dicke besitzt (Fig. 18).

15. Dass derselbe durch Mazeration in Fibrillen zerfallen kann (Fig. 17, 25 und 33).

1) Mit Retzi us (1906) nehme ich an, dass es ausserdem eine äussere von einer dünnen Cytoplasmaschicht gebildete, Kopfmembran giebt. 
16. Dass im Gebiete des Verbindungs- (oder Mittel-)stückes der Schwanzfaden von einer Cytoplasmahülle umgeben wird, welche im allgemeinen ein körniges und nur selten ein spiraliges Aussehen hat (Fig. 16, 21). (Die Långe dieser Körnchenbülle ist etwa die Hälfte der Kopflänge).

17. Dass die bei längerer Wasserbehandlung krampfartig gebogenen oder eingerollten Spermien (Fig. 30 und 31) soviel ich bisher habe finden können - sich nicht wieder strecken können und also offenbar nicht mehr befruchtungsfähig sind, obgleich sie noch mehrere Stunden lebhaft umherschwimmen können.

18. Dass die Spermien von Rana esculenta, Rana arvalis und Rana mugiens einander nicht vollständig ähnlich sind (vergl. Fig. 32-37); dass sie aber einen gemeinsamen Typus darstellen, welcher von demjenigen der Rana-fuscaSpermien stark abweicht.

19. Dass die Spermien von Rana esculenta, Rana arvalis und Rana mugiens alle eine kurze Spiralh ülle besitzen (Fig. 32, 35-37).

20. Dass sie auch ein Halsstück haben, von etwa demselben Aussehen wie dasjenige der Spermien von Rana fusca, Bombinator und Pelobates (vergl. Broman, 1900: 1, 1901: 2).

21. Dass das Perforatorium der Rana-arvalis-Spermien knopfförmig (Fig. 35) - wie dasjenige der Rana-esculentaSpermien - und dass das Perforatorium der Rana-mugiensSpermien mützenfömig ist (Fig. 37).

Lund, Dezember 1906. 


\section{Literaturverzeichnis.}

(Die mit * bezeichneten Arbeiten waren mir nicht zugänglich.)

Ankermann (18507): Einiges über die Bewegung und Entwicklung der Samenfäden des Frosches. Zeitschr. f. wiss. Zool., Bd. 8, p. 129.

Ballowitz (1890): Untersuchungen über die Struktur der Spermatozoen. Arch. f. mikr. Anat., Bd. 36.

Benda, C. (1898): Über die Spermatogenese der Vertebraten und höherer Evertebraten. Verh. der physiol. Gesellsch. zu Berlin. Jahrg. 1897-98, No. 14-17.

D erselbe (1903): Die Mitochondria. Ergebnisse der Anat. u. Entwicklungsgeschichte, Bd. XII, p. 761.

Bertacchini, Piètro (1891): La Spermatogenesi nella Rana temporaria. Internat. Monatsschr. f. Anat. u. Physiol., Bd. 8, p. 140.

Derselbe (1896): Ricerche biologiche sulla Spermatogenesi nel Gruppo degli Anfibi anuri. Ebenda, Bd. 13, p. 409.

Broman, Ivar (1900: 1): Über Bau und Enwicklung der Spermien von Bombinator igneus. Anat. Anz., Bd. 17, p. 129.

Derselbe (1900:2): Bidrag till kännedomen om batrachiespermiernas byggnad. Lund.

Derselbe (1901: 1): Über gesetzmässige Bewegungs- und Wachstumserscheinungen (Taxis- und Tropismenformen) der Spermatiden, ihrer Centralkörper, Idiozomen und Kerne. Arch. f. mikr. Anat., Bd. 59, p. 106.

Derselbe (1901: 2): Notiz über das ${ }_{\text {Halsstück }}$ der Spermien von Pelobates fuscus nebst kritischen Bemerkungen über die Nomenklatur der Spermienschwanzfäden. Anat. Anz., Bd. 20, p. 347.

Derselbe (1902): Über Bau und Entwicklung von physiologisch vorkommenden atypischen Spermien. Anat. Hefte I, Bd. 18, p. 508 .

$\mathrm{Cz}$ ermak, Jos. Jul. (1833): Beiträge zu der Lehre von den Spermatozoen. Wien 1833.

*D u val (1880): Recherches sur la Spermatogénèse chez la Grenouille. Rev. des Sc. nat. Cit. nach Bertacchini (1896).

E cker, A. (1851-1859): Icones physiologicae. Taf. XXI, Fig. Vc., Leipzig.

Gaup p, Ernst (1904): Eckers and Wiedersheims Anatomie des Frosches. III. Lehre von den Eingeweiden etc., p. 308, Braunschweig.

*Gleichen (1788): Abhandlung über die Samen- und Infusionstierchen. Nürnberg. Cit. nach Czermak (1833).

Gruenhagen, A. (1885: 1) : Untersuchungen über Samenentwicklung. Centralblatt f. med. Wiss.; Jahrg. 23, No. 28.

Derselbe (1885: 2): Über die Spermatogenese bei Rana fusca (temporaria). Ebenda No. 42.

*de l'Isle, A. (1873): Hybridation chez les Amphibies. Annales des Science nat., Sér. V, Vol. 17, p. 6. Cit. nach Pflüger (1883).

Kölliker, A. (1847): Die Bildung der Samenfäden in Bläschen als allgemeines Entwicklungsgesetz. Neue Denkschr. d. Allgem. schweiz. Gesellschaft f. d. Naturw., Bd. 8. 
Leeuwenhoek (1687): Anatomia, seu interiora rerum etc. Lugd. Batav, p. 51 in Anat. et contempl.

Leydig, Franz (1877): Die anuren Batrachier der deutschen Fauna. Bonn.

Massart (1888): Sur l'irritabilité des spermazoïdes de la grenouille. Bullet. de l'acad. roy. de Belgique, T. 18, p. 750 .

Meves, Fr. (1897): Über Struktur und Histogenese der Samenfäden von Salamandra maculosa. Arch. f. mikr. Anat., Bd. 50.

Derselbe (1898): Über das Verhalten der Centralkörper bei der Histogenese der Samenfäden von Mensch und Ratte. Verh. d. anat. Ges. in Kiel.

Derselbe (1899): Über Struktur und Histogenese der Samenfäden des Meerschweinchens. Arch. f. mikr. Anat., Bd. 54.

Derselbe (1902): Struktur und Histogenese der Spermien. Ergebnisse d. Anat u. Entwicklungsgesch., Bd. 11.

Neumann, E. (1868): Entwicklung der Samenfäden beim Frosche. Zentralbl. f. d. med. Wiss., Nr. 24, p. 369 .

Derselbe (1875): Untersuchungen über die Entwicklung der Spermatozoiden. Arch. f. mikr. Anat., Bd. 11, p. 292.

Pflüger, E. und Smith, William J. (1883): Untersuchungen über Bastardierung der anuren Batraehier und die Prinzipien der Zeugung, II. Pfiügers Archiv f. d. ges. Physiologie, Bd. 32, p. 519.

Prévost et Dumas (1824): Observations relatives à l'appareil générateur des animaux mâles; examen des liquides renfermés dans les diverses glandes qui peuvent s'y rencontrer; histoire et description des animalcules spermatiques. Annales des sciences naturelles, T. I, p. 274.

Remak, R. (1854): Über Eihüllen und Spermatozoen. Archiv f. Anat., Phys. u. wiss. Med., p. 252.

Retzius, Gustaf (1906): Die Spermien der Amphibien. Biologische Untersuchungen, N. F., Bd. 13, Stockholm u Jena, p. 49.

Schweigger-Seidel, F. (1865): Über die Samenkörperchen und ihre Entwicklung. Arch. f. mikr. Anat., Bd. 1, p. 309.

Treviranus, G. R. (1816): Über die organischen Elemente des tierischen Körpers. Vermischte Schriften anatomischen a. physiologischen Inhalts. Bd. 1, p. 122. Göttingen.

v. la Valette St. George (1871): Der Hoden. Strickers Handbuch der Lehre von den Geweben des Menschen u. der Tiere, Bd. I, p. 541. Leipzig.

Derselbe (1876): Über die Genese der Samenkörper. Vierte Mitteilung. Arch. f. mikr. Anat., Bd. 12, p. 797.

Derselbe (1886): Spermatologische Beiträge. Dritte Mitteilung. Ebenda, Bd. 27, p. 385.

Wagner und Leuckart (1852): Semen. Todds Cyclopædia of Anatomy and Physiology, Vol. 4. London.

Waldeyer, W. (1901, 1902): Die Geschlechtszellen. Hertwigs Handbuch d. vergl. u. exper. Entwicklungslehre der Wirbeltiere. Jena. 


\section{Erklärung der Abbildungen auf Tafel XIX und XX.}

Die Figuren sind mit Zeiss' Apochromat $2 \mathrm{~mm}$ (Apertur 1,30) und Kompensationsokular 12 unter Benutzung des Abbéschen Zeichenapparates (Projektion auf Objekttischhöhe. Tubuslänge $160 \mathrm{~mm}$ ) entworfen. Vergrösserung also etwa $1500 \mathrm{Mal}$.

Die Figuren 1-15 stammen alle von Präparaten, die mit dem Her mannschen Gemisch fixiert und mit Eisenhämatoxylin nach M. Heidenhain gefärbt sind.

Die Figuren 16--37 sind nach Ausstrichspräparaten, welche in verschiedener Weise behandelt wurden (vergl. unten!), gezeichnet.

Fig. 1-6. Spermatiden von Rana fusca aus der ersten Entwicklungsperiode. Fig. 7-11. Spermatiden (desselben Tieres) aus der zweiten Entwicklungsperiode.

Fig. 12 a. 13. Spermatiden (desselben Tieres) aus der dritten Entwicklungsperiode (= unreife Spermien).

Fig. 14 u. 15. Degenerierende Spermatiden, deren Centriolderivate und Mitochondrien sich in den Stadien der zweiten bezw. dritten Entwicklungsperiode befinden.

Fig. 16. Unreife Spermie von Rana fusca. Frisch mit Gentianaviolett gefärbt und nachher langsam eingetrocknet. - Kopf gequollen. Dasselbe ist teilweise auch mit dem Spiess der Fall.

Fig. 17 u. 18. Isolierte Spermienschwänze von Rana fusca. In Fig. 17 ist die abgebrochene kaudalste Kopfpartie auch sichtbar. Körnchenhülle gequollen, teilweise homogen, teilweise von spiraligem Aussehen. Von den Centriolkörnern geht mitten durch die Körnchenhülle ein einfacher Schwanzfaden aus, welcher in diesem Falle (ansnahmsweise) weiter kaudalwärts in zwei Fäden zerfällt. In Fig. 18 ist die Körnchenhülle wegmazeriert. Von den hierdurch vollständig enthüllten Centriolkörnern geht ein einfacher gleichdicker Schwanzfaden aus. - Fig. 17 stammt aus einem gewöhnlichen Trockenpräparat, welches nach dem Eintrocknen mit Gentianaviolett gefärbt wurde; Fig. 18 aus demselben Präparat wie Fig. 16.

Fig. 19. Reife Spermie von Rana fusca. - In Osmiumdämpfen fixiert und mit Gentianaviolett gefärbt. Kopf nur sehr wenig gequollen.

Fig. 20a. Reife Spermie von Rana fusca. - Bebandlung wie für Fig. 16 angegeben wurde, jedoch mit einer etwas schnelleren Eintrocknung. - Kopf stark gequollen. Verbindungsstück von homogenem Aussehen, stark gefärbt. Spiess (ausnahmsweise) an der konvexen Kopfseite.

Fig. 20b. Vordere Hälfte eines gequollenen Spermienkopfes (R. fusca) aus einem gewöhnlichen Trockenpräparat. Der Spiess ist (ausnahmsweise) en face zu sehen.

Fig. 21. Reife Spermie von Rana fusca. Durch dieselbe Behandlung, wie für Fig. 16 angegeben wurde, sind Spiess und Centriolkörner etc. deutlich geworden, obgleich in diesem Falle keine merkbare Quellung 
der Spermie eingetreten ist. - Ich kann daher diese Figur als Norm für den Bau der Rana fusca-Spermien aufstellen.

Fig. 22. Nicht ganz reife Spermie von Rana fusca. Ungefärbt, lebend beobachtet und gezeichnet. Ein Cytoplasmaballen haftet noch am Schwanzfaden.

Fig. 23. Ähnliche Cytoplasmaballen, von den Spermien abgeschnürt. Lebend gezeichnet.

Fig. 24. Kopf und Verbindungsstïck einer Rana fusca-Spermie. - Zenker Gemisch. - Eisenhämatoxylin.

Fig. 25a. Spermie von Rana fusca; frisch mit Gentianaviolett gefärbt.

Fig. 25b. Dieselbe Spermie, 18 Stunden später (nach langsamer Eintrocknung).

Fig. 26. Kopf (stark deformiert) und Verbindungsstück einer Spermie von Rana fusca. - Gewöhnliches Troekenpräparat. Gentianaviolett

Fig. 27 u. 28. Vordere Kopfpartien von Rana fusca-Spermien, deren hintere Kopfpartien körnig zerfallen sind. - Harris' Hämatoxylin.

Fig. 29. Vordere Partie eines beim Ausstreichen stark in die Länge ausgezogenen Spermienkopfes. - Rana fusca. - Gewöhnliches Trockenpräparat. Gentianaviolett.

Fig. 30 u. 31. Spermien (von Rana fusca), deren Köpfe sich eingerollt oder ösenartig zurückgebogen haben. Vier Stunden nach Wasserzusatz zu dem frischen Präparat mit Gentianaviolett gefärbt und unmittelbar danach gezeichnet.

Fig. 32-34. Spermien von Rana esculenta. - Fig. 32. Behandlung wie für Fig. 16 angegeben wurde. Fig. 33. Gewöhnliches Trockenpräparat. Fig. 34 in Osmiumdämpfen fixiert. - Alle mit Gentianaviolett gefärbt.

Fig. 35. Spermie von Rana arvalis. Frisch, mit Gentianaviolett gefärbt und unmittelbar danach abgezeichnet.

Fig. 36 a. 37 . Spermien von Rana mugiens. In Osmiumdämpfen fixiert und mit Gentianaviolett gefärbt. 